\title{
Plasmonic characteristics of rhodium dual broken nanorings in UV-visible regime
}

\author{
Yue Zhang ${ }^{1}$, Dandan Dong ${ }^{1}$, Tao Xiong ${ }^{1,2}$, Wei Wang ${ }^{1}$, Cheng Sun ${ }^{1,2, *}$ \\ ${ }^{1}$ College of Physical Science and Technology, Dalian University, 116622, China \\ ${ }^{2}$ Liaoning Engineering Laboratory of Optoelectronic Information Technology, Dalian, \\ 116622, China
}

\begin{abstract}
This work reports on the plasmonic properties of a symmetry-breaking system consisting of rhodium dual broken nanorings, in the ultraviolet-visible regime. In the structure, two rhodium broken rings are located with a separation on the scale of nanometers. As the separation, the light polarization, and the relative orientation of the broken angles are respectively varied, the plasmonic scattering efficiency of the system is investigated, using the finite difference time domain method. Multiple plasmonic resonances are revealed, and the associated asymmetry-induced Fano-like lineshapes are fitted to a model that employs multiple Fano lineshape functions. The resonance wavelengths, the spectral widths, and the characteristic $q$ values are determined from the best fit parameters, and the plasmonic characteristics of the system are quantitatively probed. The results in this work may be beneficial in designs of plasmonic devices that operates at ultraviolet-visible wavelengths.
\end{abstract}

Keywords: Plasmonics; Rhodium nanorings; Ultraviolet-visible; Finite difference time domain

${ }^{*}$ Corresponding author.

Email address: suncheng@dlu.edu.cn(Cheng Sun). 


\section{Introduction}

The plasmonic properties of noble metal-based nanostructures are mainly determined by the oscillations of their free electrons that are excited by the irradiation of incident light[1]. Surface plasmons have attracted much attention due to their ability to alter photon interactions at nanoscale and their various applications, such as optical waveguides, nonlinear optics, as well as surface-enhanced spectroscopy, etc. $[2,3,4]$ Compared with structures with certain symmetry, symmetry-breaking systems provide us with great plasmonic tunability, thanks to its non-uniform electromagnetic fields, and the induced multiple plasmonic modes[5]. To date, symmetry-breaking structures have been shown to have useful optical properties and a wide range of applications $[6,7]$.

One of the characteristics in symmetry-breaking systems is the generation of asymmetric lineshapes once the resonance condition is met, which is known as Fano resonance. The asymmetric mechanism in Fano resonance was first addressed by Ugo Fano from the perspective of quantum mechanics in 1961[8]. In general quantum systems, Fano resonance may occur when there is interference between discrete and continuous quantum states. In plasmonic nanostructures, Fano resonance may arise from the strong coupling between the discrete and the continuous mode, which manifests itself into an asymmetric lineshape in the spectrum $[9,10,11]$. Fano lineshapes can thus be adjusted by changing the parameters in plasmonic nanostructures, through affecting the coupling of the plasmonic modes[12]. For example, multiple Fano resonances were generated due to the interference of the dimer plasmonic modes in a split nanoring dimer[13]. In addition, it was also found that the symmetry breaking in a concentric ring/disk cavity enabled the coupling between plasmonic modes of differing multipolar order, which could result in a tunable Fano resonance[14, 15].

Nowadays, most plasmonic studies have been focused on metals of gold, silver and copper, etc., which respond mainly in the visible and near-infrared wavelength region[16]. However, as the investigations of novel materials keep going, the plasmonic devices and structures that operate in the ultraviolet (UV) spectrum are also in demand[17]. Therefore, it is necessary to find new metals that can be used in UV plasmonics. Among many promising UV plasmonic metals including chromium, titanium, indium, tin, magnesium, lead, and thallium, etc., rhodium has recently been shown to possess good UV plasmonic properties by both experiments and theories[18, 19]. Exper- 
imentally, it was reported that the surface plasmon resonances in the UV region could be tuned by controlling the size and morphology of rhodium nanoparticles[20]. Tunable optical features at UV wavelengths were also addressed in self-organized rhodium nanostructures[21]. In theory, the UV plasmonic behavior and surface charge distribution of rhodium tetrahedra were studied, and the applications in surface-enhanced spectroscopies and photocatalysis were discussed[22]. In addition, rhodium nanoparticles of planar tripods were investigated and the potential use in plasmonically enhanced UV photocatalysis was also demonstrated[23].

In this work, a symmetry-breaking system comprised of rhodium dual broken nanorings was proposed, and the associated plasmonic characteristics in the UV-visible regime were investigated. Multiple asymmetric lineshapes are revealed in the frequency dependent scattering efficiency curve, correlating to multiple Fano resonances. The electric field distributions at resonance wavelengths were presented, and detailed quantitative studies were also carried out.

\section{Structure and method}

The structure of rhodium dual broken nanorings is illustrated in Fig.1. In this work, the centers of both rings were placed along the $x$-axis, and their $y$ and $z$ coordinates were always kept the same, respectively. For simplicity, the sizes of the rings were set the same, i.e., the outer radius was $R=10 \mathrm{~nm}$, the inner radius was $r=8 \mathrm{~nm}$, the thickness was $h=10 \mathrm{~nm}$, and the broken angle was $\theta=30^{\circ}$. The separation (labeled ' $d$ ') was defined as the distance between the centers of the rings, and the value of $d$ was chosen to be greater than $20 \mathrm{~nm}$, to make sure the two rings were separate from each other. Two scenarios were studied in this work: as indicated in Fig.1a, the positions of the broken parts were placed in a way that they faced each other, and it formed a mirror-image like geometry along the $x$-axis. In Fig.1b, the left rhodium broken ring was fixed, while the ring on the right hand side was rotated about the $z$-axis, and the rotation angle (labeled ' $\psi_{r}$ ') represents the clockwise-rotating angle, with respect to its original position.

In this work, numerical simulations were carried out by employing the finite difference time domain (FDTD) method[24]. A plane-wave light was incident along the $z$-axis, in the UV-visible wavelength range of $130-600 \mathrm{~nm}$. The polarization of the light was denoted by the angle that was away from the $x$-axis, that is, $\varphi_{P}=0^{\circ}$ indicates the $x$-polarized light, and it becomes $y$ - 

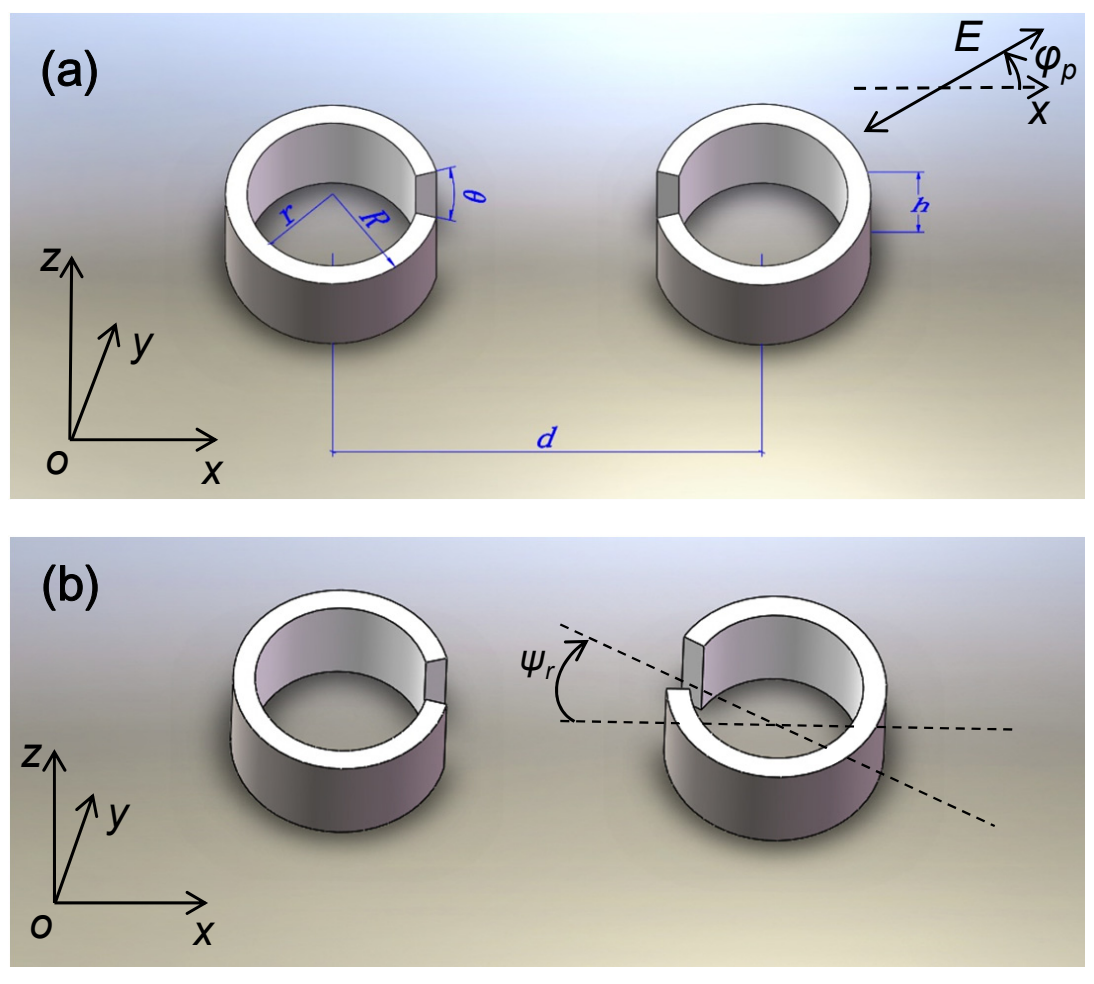

Figure 1: Schematic of the structure of rhodium dual broken nanorings. The sizes of two rings are the same. The outer radius of the ring is labeled ' $R$ ', the inner radius is indicated in ' $r$ ', , the thickness is denoted ' $h$ ', and the broken angle is labeled ' $\theta$ '. The centers of both rings are along the $x$-axis, and the $y$ and $z$ coordinates are the same, respectively. The separation is indicated in ' $d$ ', which is the distance between the centers of the rings. (a) a mirror-image like geometry along the $x$-axis, where the positions of the broken parts are faced each other. The polarization of the incident light is denoted by the angle ' $\varphi_{P}$ ', which is the angle between the electric vector and the $x$-axis. (b) the left ring is fixed, while the right ring is rotated about the $z$-axis, and the rotation angle is labeled ' $\psi_{r}$ ', which represents the clockwise-rotating angle starting from its original position. 

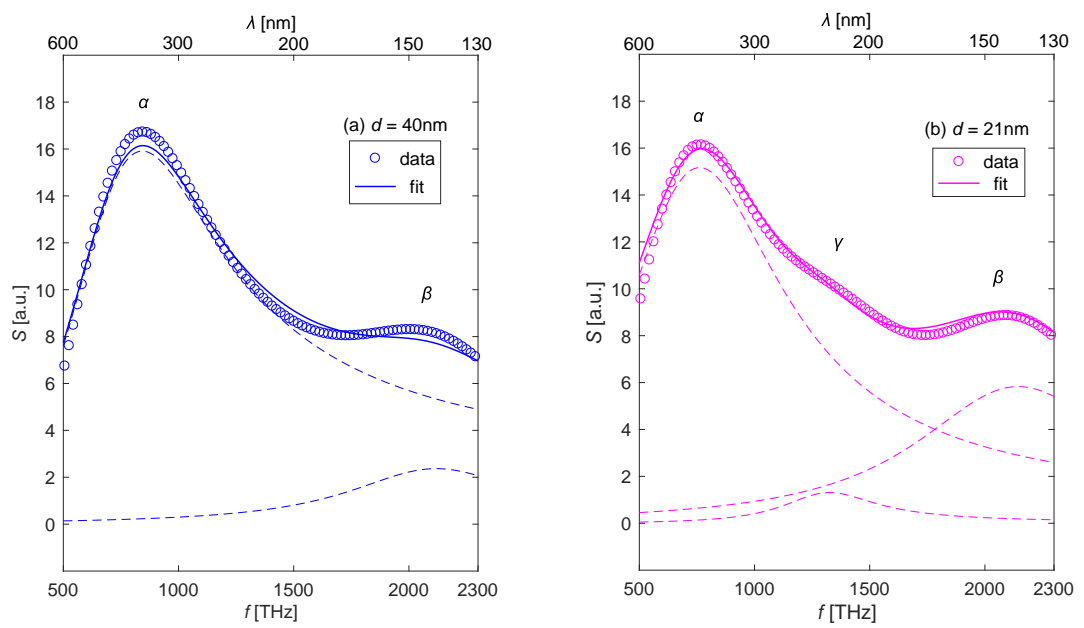

Figure 2: Frequency-dependent scattering efficiency (denoted ' $S$ ') of the structure shown in Fig.1a. (a) $d=40 \mathrm{~nm}$, and (b) $d=21 \mathrm{~nm}$. The incident light was $x$-polarized. The solid curve in (a) represents a best fit to Eq.2, and the one in (b) is a best fit to Eq.3. Dashed curves are individual components in each fit.

polarized when $\varphi_{P}=90^{\circ}$. In the simulation, perfect matching layer (PML) boundary conditions were used in all directions. The optical constants of rhodium were from Palik's experimental data[25].

\section{Results and discussions}

According to the structure shown in Fig.1a, the scattering efficiency was first computed, and two exemplary results are presented in Fig.2. In Fig.2a the separation was as great as $40 \mathrm{~nm}$, whereas in Fig.2b the separation was small, that is, $d=21 \mathrm{~nm}$. In both figures, all other parameters were kept the same, and their values were mentioned above. As shown in Fig.2a, two distinguishable peaks are observed in the scattering efficiency curve, indicating two plasmonic resonances. One occurs at the UV-visible wavelength of about $355 \mathrm{~nm}$, and the other appears at the much shorter UV wavelength of around 140nm. Both resonances display asymmetric lineshapes, and this is due to the symmetry-breaking structure of the rhodium broken rings.

As known to the community, Fano resonance may take place in symmetrybreaking systems, when the interference between a continuum and a discrete state of a quantum system is established, and the asymmetric lineshape may 
quantitatively be described by the Fano function, in which the degree of the asymmetry can be characterized by its $q$ factor[8].

$$
\frac{(\epsilon+q)^{2}}{\epsilon^{2}+1}
$$

In Eq. 1 the reduced energy variable may be written as $\epsilon=\left(f-f_{0}\right) / f_{s}$, where $f$ is the frequency of the incident light, $f_{0}$ is the resonance frequency, and $f_{s}$ denotes the spectral width of the discrete state. The characteristic parameter, $q$, depends on both the continuum and the discrete states of the quantum system.

Generally, in plasmonic systems where multiple discrete states exist, multiple Fano resonances may occur. Regarding Fig.2a, the double-peak feature in the scattering efficiency curve is a manifestation of two Fano resonances, and hence the scattering efficiency (indicated in ' $S$ ') may be fitted to Eq.2, which consists of two Fano lineshape functions.

$$
S=A_{\alpha} \frac{\left(\epsilon_{\alpha}+q_{\alpha}\right)^{2}}{\epsilon_{\alpha}^{2}+1}+A_{\beta} \frac{\left(\epsilon_{\beta}+q_{\beta}\right)^{2}}{\epsilon_{\beta}^{2}+1}
$$

where $A_{\alpha}$ and $A_{\beta}$ are coefficients. The best fit result is also shown in Fig.2a as the solid curve, which is in good agreement with the simulated data. This shows that in the region of $130-600 \mathrm{~nm}$, the plasmonic properties of the system can be decomposed into two individual Fano resonances (labeled $\alpha$ and $\beta$, respectively).

Referring to Fig.2b, apart from Peaks $\alpha$ and $\beta$, the third peak at about $230 \mathrm{~nm}$ becomes non-negligible, as the separation of the nanorings is reduced to a small value of $d=21 \mathrm{~nm}$. Note that the data in Fig.2b was first fitted to Eq.2, and yet the region around 230nm could not be well fitted using two Fano lineshapes only (for brevity, that fit is not shown here). The scattering efficiency curve was then fitted to Eq.3 by considering three Fano lineshape functions, and the best fit result is also given in Fig.2b. The consistency between the fit and the data infers that a third Fano resonance occurs at the UV wavelength of about 230nm, and it is labeled $\gamma$ in this work.

$$
S=A_{\alpha} \frac{\left(\epsilon_{\alpha}+q_{\alpha}\right)^{2}}{\epsilon_{\alpha}^{2}+1}+A_{\beta} \frac{\left(\epsilon_{\beta}+q_{\beta}\right)^{2}}{\epsilon_{\beta}^{2}+1}+A_{\gamma} \frac{\left(\epsilon_{\gamma}+q_{\gamma}\right)^{2}}{\epsilon_{\gamma}^{2}+1}
$$

To further probe the Fano resonances at different wavelengths, the distributions of the electric fields at the peak wavelengths were calculated, and the 

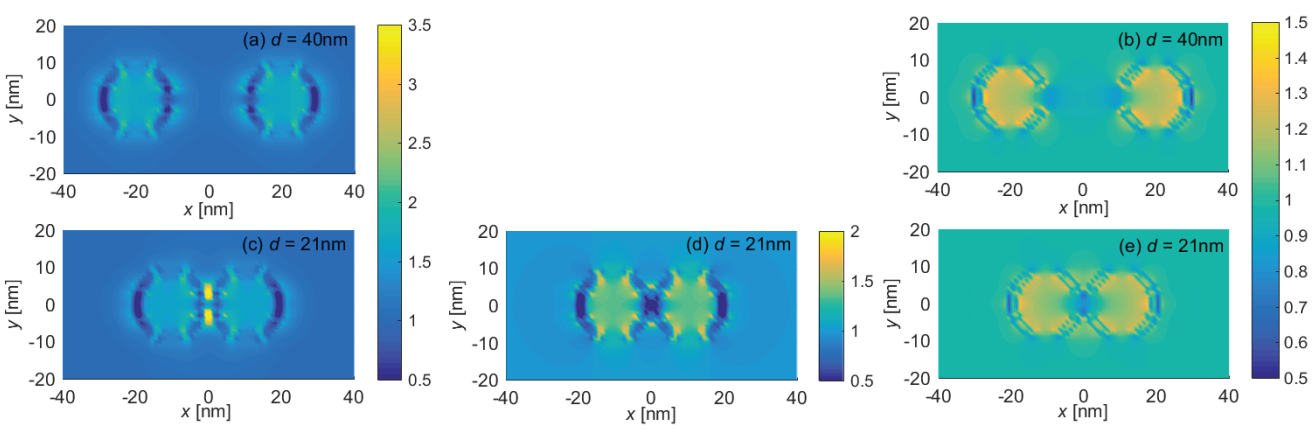

Figure 3: Electric field distribution in the $x-y$ plane for the cases shown in Fig.2. The electric fields were determined from (a)-(b) $d=40 \mathrm{~nm}$ and (c)-(e) $d=21 \mathrm{~nm}$. The wavelength correlates to (a) and (c) Peak $\alpha$; (b) and (e) $\beta$; (d) $\gamma$. In the $z$ direction, the $x-y$ plane was through the center of the rhodium rings. Note that the maximum intensity of the color bar is not the same.

results are plotted in Fig.3. By comparing Fig.3a and 3b, it is evident that Peaks $\alpha$ and $\beta$ in Fig.2a arise from two different electromagnetic modes. By examining Fig.3a and 3c, it is shown that as the rhodium rings' separation is narrowed, the electromagnetic hot spots (i.e., the electric field with great intensity) appear more pronounced in the middle space between the broken rings. This effect has been observed in many other nanostructures and it can also be utilized as a way to generate practical hot spots. Besides, the similarity between Fig.3c and Fig.3d reveals that Peaks $\alpha$ and $\gamma$ in Fig.2b undergo similar physical mechanisms, albeit the intensities are different.

Systematically, the effects of the separation on the scattering efficiency curve were studied, with changing $d$ from $60 \mathrm{~nm}$ to $21 \mathrm{~nm}$. The results are plotted in Fig.4. Note that the best fit curves shown in Fig.4a were obtained by using Eq.2, while those in Fig.4b were from Eq.3. This indicates that once the separation is as large as $30 \mathrm{~nm}$, the lineshape can be decomposed into two separate Fano resonances. However, when the separation is small enough, e.g., $23 \mathrm{~nm}$, the third resonance peak becomes obvious and cannot be neglected. To quantitatively probe the resonance properties, the best fit values for the resonance wavelength, the spectral width, and the characteristic $q$ parameter were extracted, and the corresponding data are given in Fig.5.

Regarding Fig.5a, with narrowing the separation from $60 \mathrm{~nm}$ to $21 \mathrm{~nm}$, the resonance wavelength of Peak $\alpha$ keeps almost at $435 \mathrm{~nm}$, Peak $\beta$ is constant at $140 \mathrm{~nm}$, and Peak $\gamma$ is at 225nm, if any. From Fig.5b, it is witnessed that as $d$ 

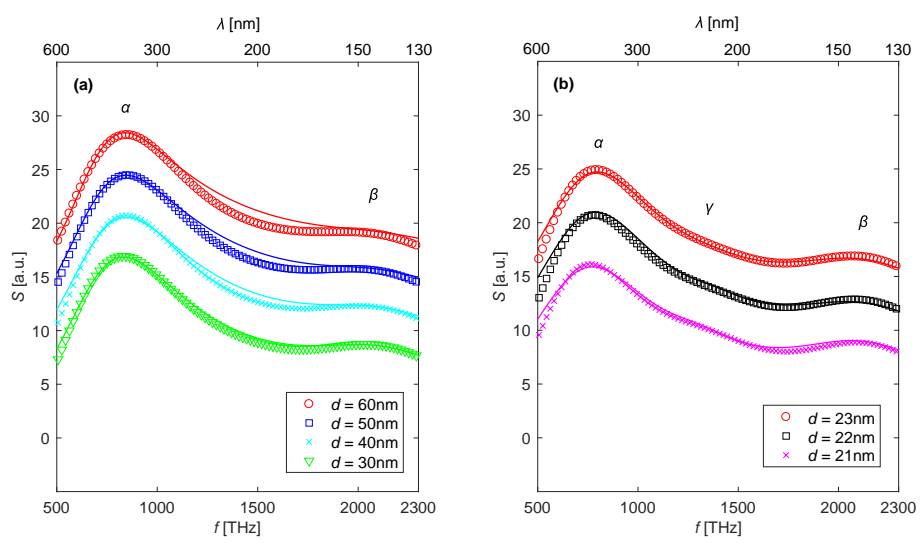

Figure 4: Frequency-dependent scattering efficiency of the structure shown in Fig.1a. (a) $d$ was from 60 to $30 \mathrm{~nm}$, and (b) from 23 to $21 \mathrm{~nm}$. The incident light was $x$-polarized. The solid curves in (a) represent a best fit to Eq.2, and the ones in (b) are a best fit to Eq.3. For clarity, the curves are presented with an offset in the $y$-axis: in (a) $d=40 \mathrm{~nm}$ is offset by 4 , the $50 \mathrm{~nm}$ the 8 , and the $60 \mathrm{~nm}$ the 12 ; in (b) $d=22 \mathrm{~nm}$ is offset by 4 , and the $23 \mathrm{~nm}$ the 8 .

is decreased, the spectral width of Peak $\alpha$ is raised from 370 to $430 \mathrm{THz}$, and Peak $\beta$ is broadened from 400 to $550 \mathrm{THz}$; Peak $\gamma$ oscillates slightly within 230 and $250 \mathrm{THz}$. Interestingly, different trends are revealed in the $q$ values that are shown in Fig.5c. With the value of $d$ is decreased, Peak $\alpha$ 's $q$ value is raised from 2 to 6 , and Peak $\gamma$ from 8 to 10 , while Peak $\beta$ is reduced from 28 to 23. This difference in the trends for $q$ values verifies the aforementioned fact that Peaks $\alpha$ and $\beta$ are associated with two different modes.

Known to the community, the polarization of the electric vector of the incident light may also affect the resonance behaviors associated with plasmonic systems, since the surface plasmons are induced by the incident light with a certain polarization. In this work, the effect of the light's polarization was also studied, by varying the polarization angle from 0 to $90^{\circ}$. Taking the structure of $d=40 \mathrm{~nm}$ as an example, the polarization angle dependent scattering efficiency curves were simulated and the results are shown in Fig.6. Based on the data given in Fig.6, the curves were all fitted to Eq.2, and the best fit results are presented as solid curves in the figure. It is clear that the resonance peaks $\alpha$ and $\beta$ can both be well fitted for all the polarization angles. In addition, the field distributions at the resonance wavelengths were also computed, and the results are illustrated in Fig.7. For both Fig.7a and $7 \mathrm{~b}$, as $\varphi_{P}$ is changed, it is obvious that the spatial regions where the 

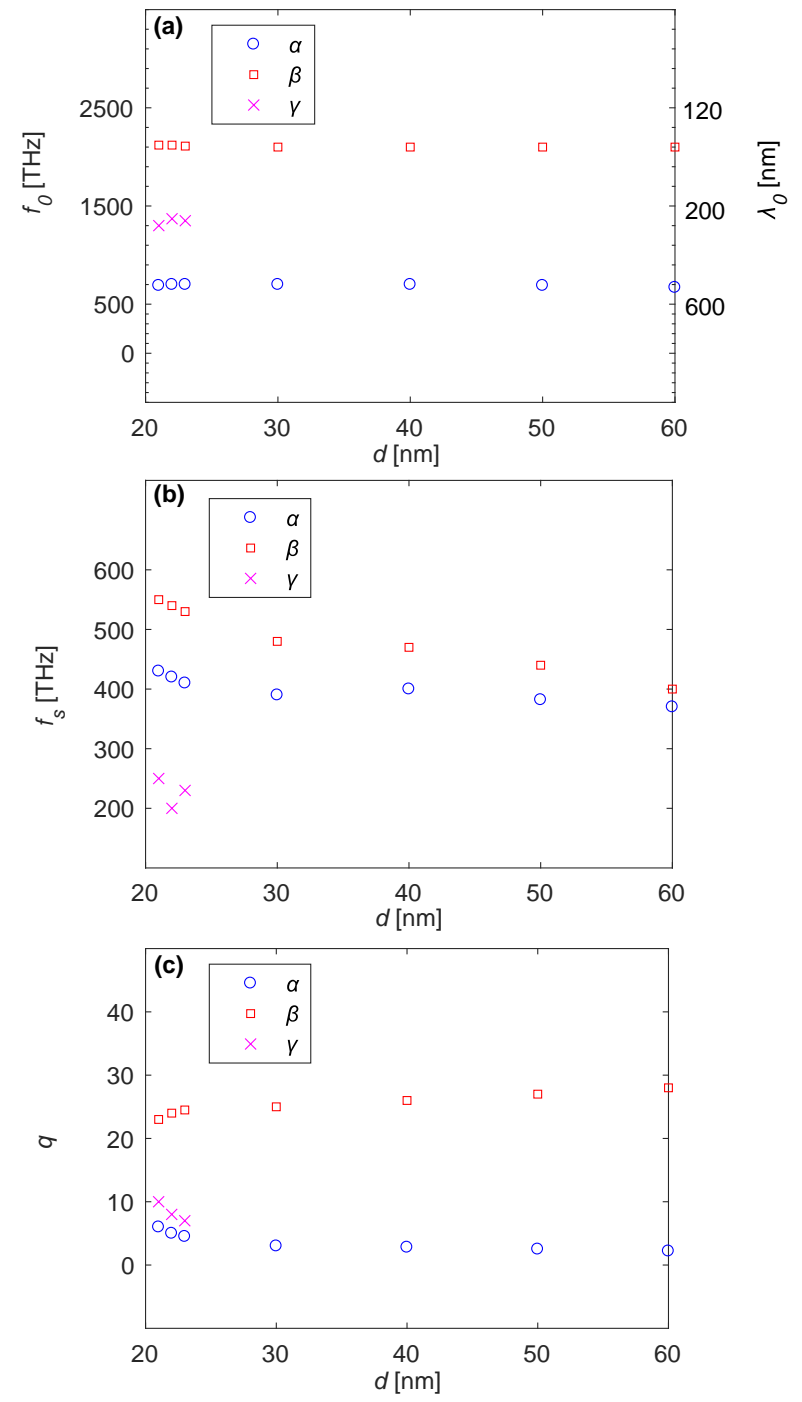

Figure 5: Best fit values for (a) resonance frequency/wavelength, $f_{0} / \lambda_{0}$, (b) spectral width, $f_{s}$, and (c) $q$ parameter, as a function of the separation, $d$, determined by fitting the scattering efficiency curve in Fig.4. 


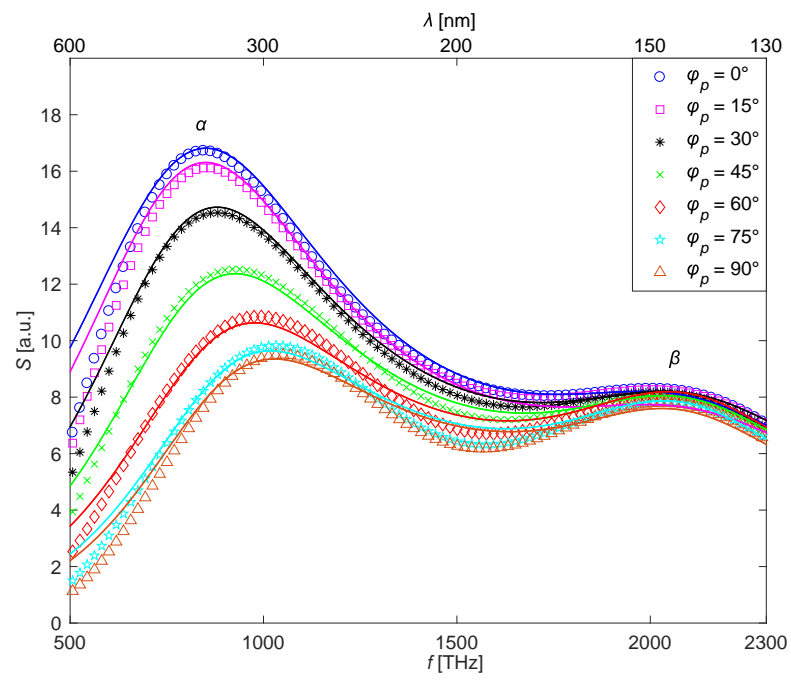

Figure 6: Frequency-dependent scattering efficiency of the structure shown in Fig.1a, with $d=40 \mathrm{~nm}$. The polarization of the incident light was varied from $\varphi_{P}=0$ to $90^{\circ}$. The solid curves are a best fit to Eq.2.

hot spots appear strongly depend on the polarization of the incident light. Similar effects were also observed and addressed in previous work[26].

Based on Fig.6, the best fit parameters for Peaks $\alpha$ and $\beta$ were extracted, and the values are presented in Fig.8. As indicated in Fig.8a, with changing the polarization vector from the $x$-axis to the $y$-axis (i.e., $\varphi_{P}$ from 0 to $90^{\circ}$ ), the resonance wavelength of Peak $\alpha$ is blueshifted from 400 to $350 \mathrm{~nm}$, while Peak $\beta$ remains unchanged at $150 \mathrm{~nm}$. Referring to Fig.8b, the spectral widths for the two peaks are both reduced, that is, the spectral width of Peak $\alpha$ is decreased from 450 to $410 \mathrm{THz}$, and Peak $\beta$ from 530 to $430 \mathrm{THz}$. Regarding Fig.8c, with increasing the value of $\varphi_{P}$, the $q$ parameter of Peak $\alpha$ is slightly decreased from 6 to 3 , whereas Peak $\beta$ is increased from 20 to 33 .

As mentioned above, Fano resonance usually takes place in symmetrybreaking systems, and its plasmonic properties are correlated to the degree of the asymmetry. In this work, the effects of asymmetry on the plasmonic behaviors were also probed, by considering the geometry that is demonstrated in Fig.1b. According to Fig.1b, the left rhodium broken ring was fixed, while the ring on the right-hand side was deliberately rotated about the $z$-axis, in order to vary the degree of the asymmetry by changing the location of the broken part of the ring. Under this scheme, the scattering efficiency curves 

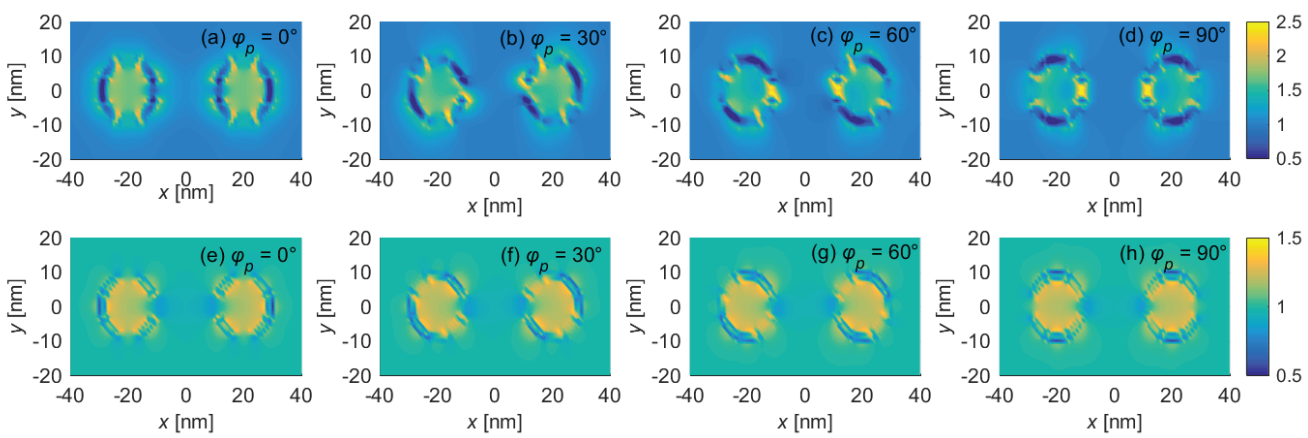

Figure 7: Electric field distribution in the $x-y$ plane for the case shown in Fig.6. The electric fields were determined at the wavelength of (a)-(d) Peak $\alpha$, and (e)-(h) $\beta$. In the $z$ direction, the $x-y$ plane was through the center of the rhodium rings. Note that the maximum intensities of the color bars in (a)-(d) and (e)-(h) are different.

were calculated, and they are plotted in Fig.9 as a function of the rotation angle of the right-hand side ring, $\psi_{r}$. An interesting fashion is witnessed from Fig.9: the curves of $\psi_{r}=0^{\circ}$ and $180^{\circ}$ coincide with each other, $\psi_{r}=30^{\circ}$ and $150^{\circ}$ are very close, and moreover, $\psi_{r}=60^{\circ}$ and $120^{\circ}$ are very similar. To probe the underlying physics of this observation, the field distributions at resonance wavelengths were further collected and are shown in Fig.10.

Regarding Fig.10, for both Peaks $\alpha$ and $\beta$, as the rotation angle, $\psi_{r}$, is changed from 0 to $180^{\circ}$, the field distributions appear similar once two rotation angles have a summation of $180^{\circ}$. For example, the field of $\psi_{r}=$ $0^{\circ}$ is quite similar to that of $180^{\circ}$, and $\psi_{r}=60^{\circ}$ has little variations with respect to that of $120^{\circ}$. To quantitatively verify this, the best fit parameters were determined for Fig.9, and the values are presented in Fig.11. It is clear from Fig.11a that little variations in the resonance wavelength are shown for both peaks, respectively; $\lambda_{0}$ is almost $390 \mathrm{~nm}$ for Peak $\alpha$, and it is around $130 \mathrm{~nm}$ for $\beta$. The observations made in Fig.11b and 11c also well support the fashion witnessed in Fig.9. For instance, The spectral width of Peak $\alpha$ for $\psi_{r}=30^{\circ}$ and $150^{\circ}$ are both $430 \mathrm{THz}$, and the $q$ value of Peak $\beta$ for $\psi_{r}=$ $60^{\circ}$ and $120^{\circ}$ are both 24 .

\section{Conclusions}

In this work, the plasmonic properties of a symmetry-breaking system consisting of rhodium dual broken nanorings have been investigated. The 

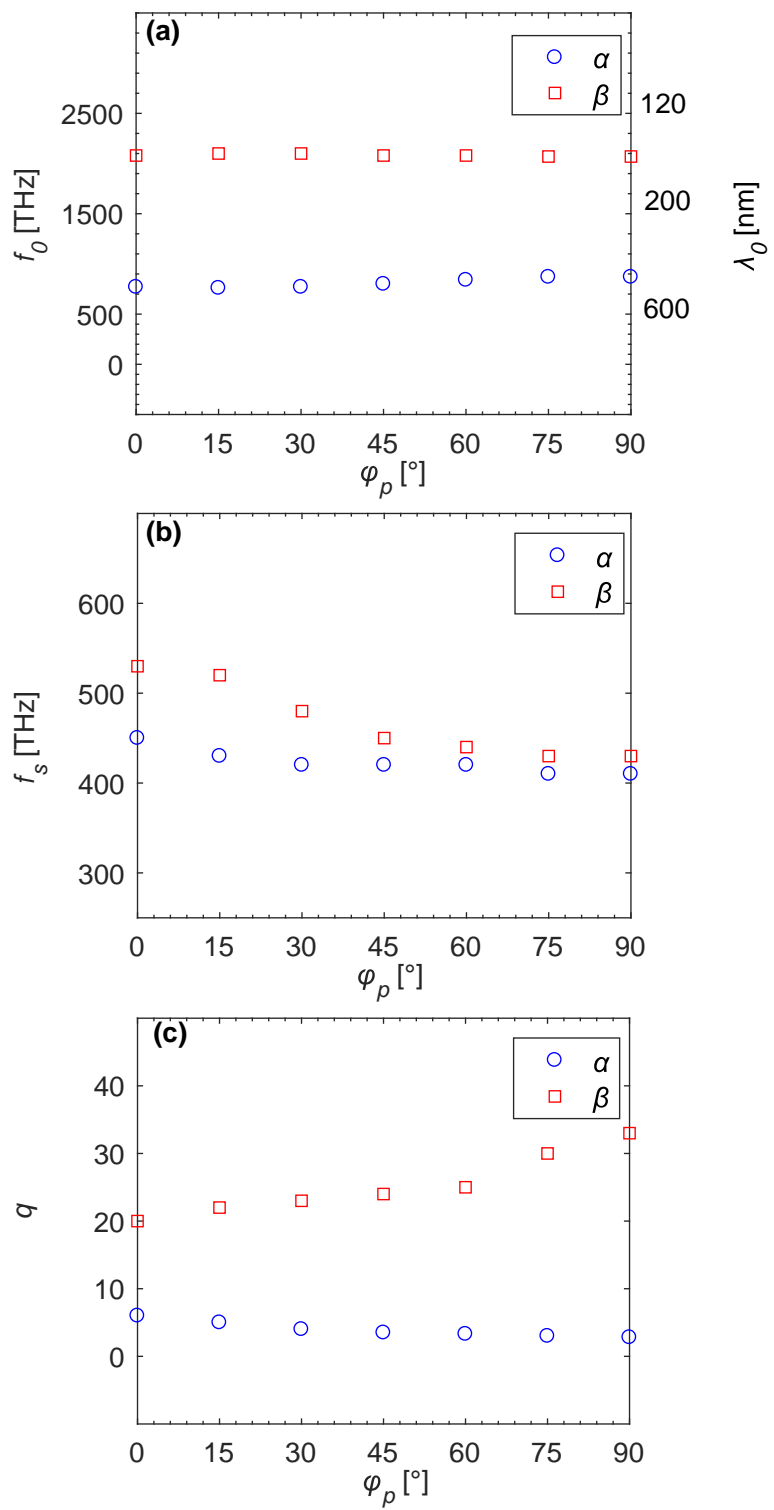

Figure 8: Best fit values for (a) resonance frequency/wavelength, (b) spectral width, and (c) $q$ parameter, as a function of the light polarization, $\varphi_{P}$, determined by fitting the scattering efficiency curve in Fig.6. 


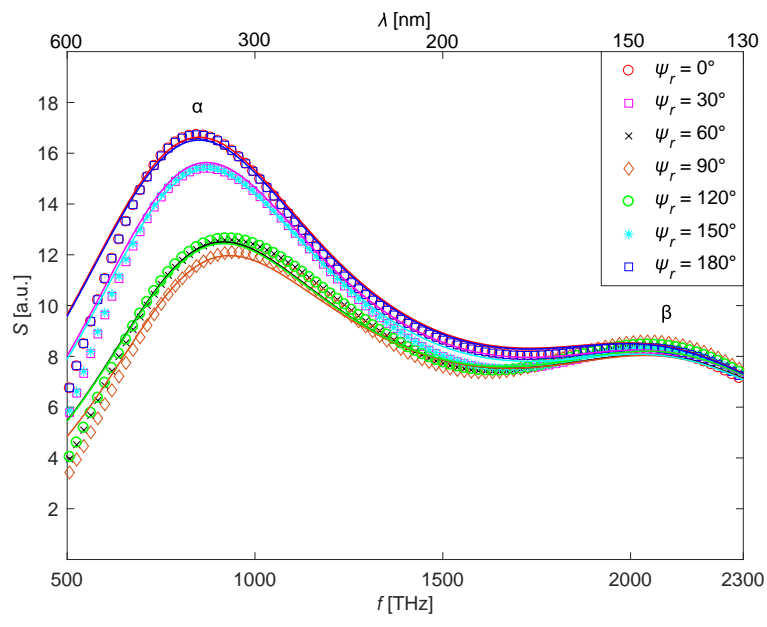

Figure 9: Frequency-dependent scattering efficiency of the structure shown in Fig.1b, with $d=40 \mathrm{~nm}$. The polarization of the incident light was $x$-polarized. The rotation angle of the right-hand side rhodium broken ring was varied from $\psi_{r}=0$ to $180^{\circ}$. The solid curves are a best fit to Eq.2.
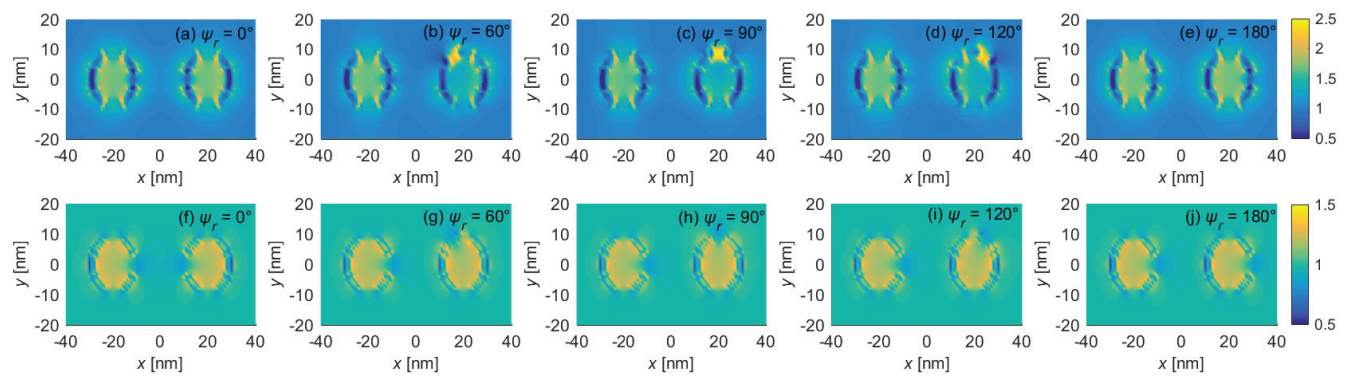

Figure 10: Electric field distribution in the $x-y$ plane for the case shown in Fig.9. The electric fields were determined at the wavelength of (a)-(e) Peak $\alpha$, and (f)-(j) $\beta$. In the $z$ direction, the $x-y$ plane was through the center of the rhodium rings. Note that the maximum intensities of the color bars in (a)-(e) and (f)-(j) are different. 

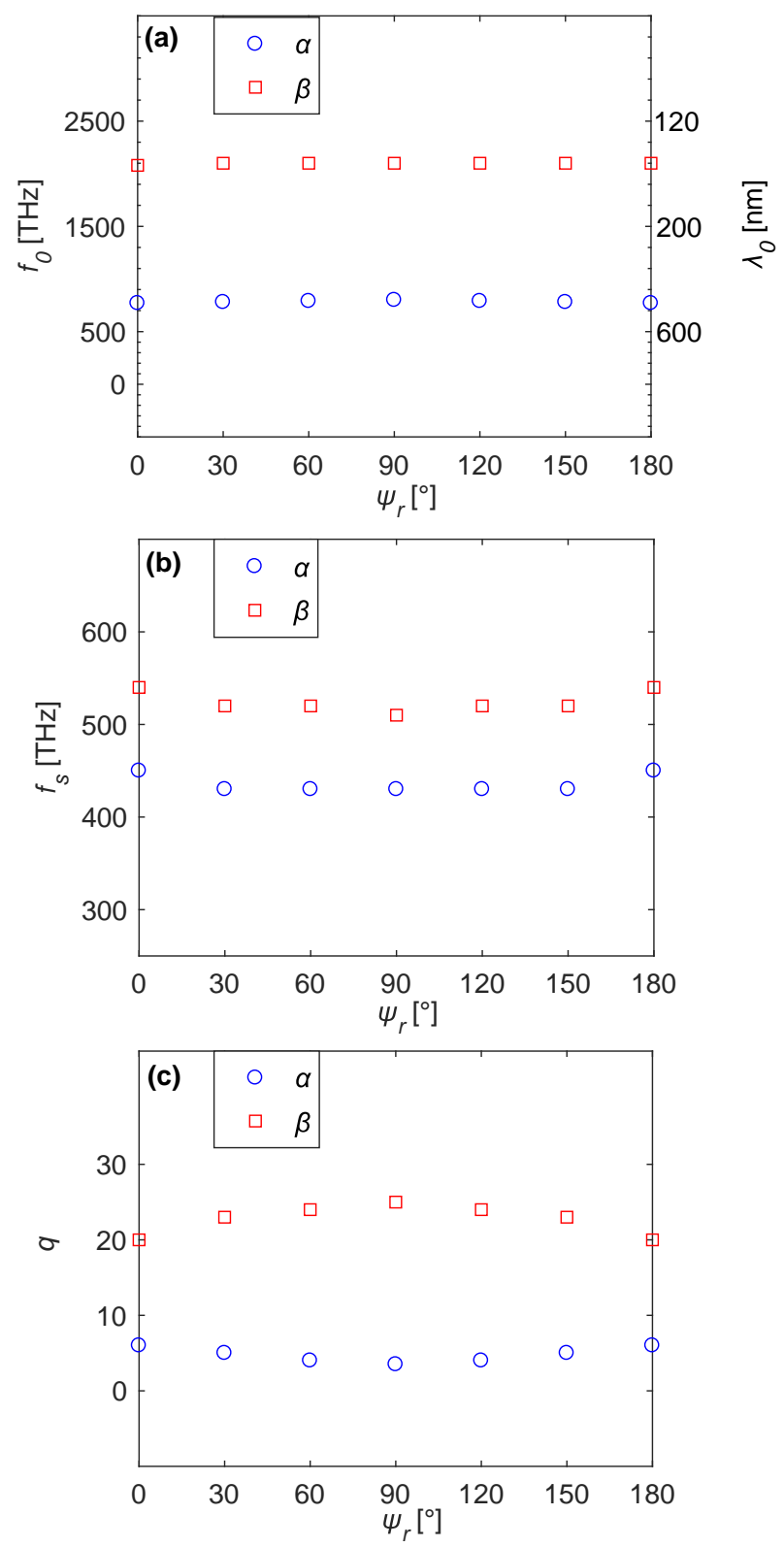

Figure 11: Best fit values for (a) resonance frequency/wavelength, (b) spectral width, and (c) $q$ parameter, as a function of the rotation angle of the right-hand side rhodium ring, $\psi_{r}$, determined by fitting the scattering efficiency curve in Fig.9. 
scattering efficiency curves have been calculated in UV-visible wavelength range, using the FDTD method. Multiple resonances have been identified and the data have been well fitted to the equations that employ multiple Fano lineshape functions. With respectively varying the separation between the broken rings, the polarization of the incident light, and the rotation angle of one of the dual rings, the plasmonic properties of the system have been studied. The electric field distributions at resonance wavelengths have also been simulated, indicating different plasmonic modes occurring at different resonances. The best fit parameters of the resonance wavelength, the spectral width, as well as the $q$ parameter have been determined, and a quantitative analysis of the system has been achieved. According to this work, rhodiumbased nanostrcutures may be implemented in designs of plasmonic devices in the UV-visible regime.

\section{Acknowledgement}

D.D. Dong acknowledges support by Grant Number 51801017 from the National Natural Science Foundation of China.

\section{Declarations}

Ethics Approval (The authors assure that this material is the authorsown original work, which has not been previously published elsewhere. The paper is not currently being considered for publication elsewhere. The paper reflects the authors own research and analysis in a truthful and complete manner. The paper properly credits the meaningful contributions of co-authors and co-researchers. The results are appropriately placed in the context of existing research. All authors have been personally and actively involved in substantial work leading to the paper, and will take public responsibility for its content.)

Consent to Participate (All authors agreed to participate in this research.)

Consent for Publication (Permission from all the authors has been taken to publish this manuscript.)

Competing Interests (The authors declare no competing interests.)

Authors' Contributions (All authors contributed to the design and implementation of the research, analysis of the results, and the manuscripts preparation.) 
Funding (D.D. Dong acknowledges support by Grant Number 51801017 from the National Natural Science Foundation of China.)

Availability of data and material (All data generated or used during the study appear in the submitted article.)

Code Availability (Code will be made available on reasonable request.) 


\section{References}

[1] Ozbay E (2006) Plasmonics: Merging Photonics and Electronics at Nanoscale Dimensions. Science. 311:189-193.

[2] Barnes WL, Dereux A, Ebbesen TW (2003) Surface plasmon subwavelength optics. Nature. 424:824-830.

[3] Kauranen M, Zayats AV (2012) Nonlinear plasmonics. Nature Photonics. 6:737-748.

[4] Yang SZ, Liu QA, Liu YL, Weng GJ, Zhu J, Li JJ (2021) Recent progress in the optical detection of pathogenic bacteria based on noble metal nanoparticles. Microchimica Acta.188:258.

[5] Kanté B, Park YS, Brien KO, Shuldman D, Lanzillotti-Kimura ND, Wong ZJ, Yin XB, Zhang X (2012) Symmetry breaking and optical negative index of closed nanorings. Nature Communications. 3:1180.

[6] Park JH, Ndao A, Cai W, Hsu LY, Kodigala A, Lepetit T, Lo YH, Kanté B (2020) Symmetry-breaking-induced plasmonic exceptional points and nanoscale sensing. Nature Physics. 16:462-468.

[7] Bereket DD, Alemayehu NK, Song XW, Lin JQ. Effect of Symmetry Breaking on Plasmonic Coupling in Nanoring Dimers[J]. Plasmonics. 2020,15(4):1977-1988.

[8] Fano U (1961) Effects of Configuration Interaction on Intensities and Phase Shifts. Physical Review. 124(6).

[9] Limonov MF, Rybin MV, Poddubny AN, Kivshar YS (2017) Fano resonances in photonics. Nature Photonics 11:543-554.

[10] Miroshnichenko AE, Flach S, Kivshar YS (2010) Fano resonances in nanoscale structures. Reviews of Modern Physics 82(3):2257-2298.

[11] Boris L, Nikolay IZ, Stefan AM, Naomi JH, Peter N, Harald G, Chong TC (2010) The Fano resonance in plasmonic nanostructures and metamaterials. Nature Materials 9(9). 
[12] Fu YH, Zhang JB, Yu YF, Luk'yanchuk B (2012) Generating and manipulating higher order Fano resonances in dual-disk ring plasmonic nanostructures. ACS nano. 6(6).

[13] Khan AD, Khan SD, Khan RU, Ahmad N, Ali A, Khalil A, Khan FA (2014) Generation of Multiple Fano Resonances in Plasmonic Split Nanoring Dimer. Plasmonics.9(5):1091-1102.

[14] Hao F, Sonnefraud Y, Dorpe PV, Maier SA, Halas NJ, Nordlander P (2008) Symmetry breaking in plasmonic nanocavities: subradiant LSPR sensing and a tunable Fano resonance. Nano Letters. 8(11):3983C3988 .

[15] Hao F, Nordlander P, Sonnefraud Y, Dorpe PV, Maier SA (2009) Tunability of subradiant dipolar and Fano-type plasmon resonances in metallic ring/disk cavities: implications for nanoscale optical sensing. ACS Nano. 3(3):643C652.

[16] Khurana K, Jaggi N (2021) Localized Surface Plasmonic Properties of $\mathrm{Au}$ and Ag Nanoparticles for Sensors: a Review. Plasmonics 16:981-999.

[17] Yael G, Rodrigo AO, Dolores O, Jos MS, Francisco G, Fernando M (2018) Plasmonics in the Ultraviolet with Aluminum, Gallium, Magnesium and Rhodium. Applied Sciences. 8(1).

[18] Ahmadivand A, Sinha R, Kaya S, Nezih P (2016) Rhodium Plasmonics for Deep-Ultraviolet Bio-Chemical Sensing. Plasmonics. 11,(3):839-849.

[19] Alcaraz OR, Sanz JM, Barreda AI, Saiz JM, Gonza?lez F, Everitt HO, Moreno F (2015) Rhodium Tripod Stars for UV Plasmonics. Journal of Physical Chemistry C. 119(22):12572-12580.

[20] Zhang X, Li P, Barreda A, Gutierrez Y, Gonzalez F, Moreno F, Everitt HO, Liu J (2016) Size-tunable rhodium nanostructures for wavelengthtunable ultraviolet plasmonics. Nanoscale Horizons. 1(1): 75-80.

[21] Bhaskar R. Sathe, Beena K. Balan, and Vijayamohanan K. Pillai (2010) Tunable optical features from self-organized rhodium nanostructures. Applied Physics Letters.96:233102.

[22] Yael G, Francisco G, Fernando M (2019) The UV Plasmonic Behavior of Rhodium TetrahedronsA Numerical Analysis. Applied Sciences. 9(19):3947. 
[23] Watson AM, Zhang X, Alcaraz OR, Marcos SJ, Gonzlez F, Moreno F, Finkelstein G, Liu J, Everitt HO (2015) Rhodium nanoparticles for ultraviolet plasmonics. Nano letters. 15(2): 1095-1100.

[24] FDTD Solutions, www.lumerical.com.

[25] Palik ED (1998) Handbook of optical constants of solids, Vol. 3, Academic press.

[26] Zhang TH, He JY, Xie W, Sun C (2020) Plasmonic properties of a honeycomb structure formed by metallic nanoparticles. Physica E. 118:113901. 